Revue d'histoire des chemins de fer

\section{Revue d'histoire des chemins de fer}

$41 \mid 2010$

Approvisionnement ferroviaire et pratiques alimentaires des citadins

\title{
Un chantier à ouvrir : l'histoire de la restauration à la SNCF. Premières orientations
}

A blueprint for the future: the history of the SNCF's food service. An introduction

\section{François Caron}

\section{(2)enEdition}

Journals

Édition électronique

URL : https://journals.openedition.org/rhcf/1208

DOI : 10.4000/rhcf.1208

Éditeur

Rails \& histoire

Édition imprimée

Date de publication : 30 avril 2010

Pagination : 227-241

ISSN : 0996-9403

Référence électronique

François Caron, « Un chantier à ouvrir : I'histoire de la restauration à la SNCF. Premières orientations », Revue d'histoire des chemins de fer [En ligne], 41 | 2010, mis en ligne le 30 avril 2012, consulté le 28 juin 2022. URL : http://journals.openedition.org/rhcf/1208; DOI : https://doi.org/10.4000/rhcf.1208 
François CARON

\section{Un chantier à ouvrir : I'histoire de la restauration à la SNCF. Premières orientations} donné d'une recherche en cours. Il pose plus de problèmes qu'il n'en résout. Les archives disponibles pour traiter ce sujet sont encore difficiles à localiser et leur richesse est très irrégulière. Les données que je vous présente aujourd'hui sont provisoires, car je suis bien conscient de n'être pas parvenu à identifier toutes les sources disponibles. Après 1976 elles se limitent à des sources imprimées, qu'il faudra inventorier ${ }^{1}$. Je laisserai de côtés les aspects culturels et sociétaux de la restauration, qui mériteraient une étude particulière. Il me semble absurde de toute manière d'entreprendre de telles recherches sans posséder une bonne connaissance des réalités techniques et organisationnelles de l'objet étudié, comme le font tant de chercheurs aujourd'hui, particulièrement en histoire des transports.

La restauration ferroviaire a toujours été pour la SNCF une source de difficultés techniques et organisationnelles fort irritantes dans un monde obsédé par la rationalisation de la gestion. Elles permettent de comprendre

1- Sous bénéfice de l'inventaire du versement de la direction commerciale de la SNCF relatif à la période 1990-2001 prévu au Centre des archives historiques (Le Mans), annoncé par L. Bour dans sa contribution au présent volume, voir p. 37 [N.d.l.R.]. 
l'extrême instabilité des solutions adoptées dans chacun des trois domaines que l'on peut distinguer :

- celui du financement des services de restauration et des relations contractuelles avec les concessionnaires de ces services,

- celui des moyens techniques mis en œuvre pour répondre aux attentes de la clientèle dans le domaine de l'organisation des services,

- celui enfin des choix dans le domaine d'une offre alimentaire adaptée, elle aussi, à ces attentes.

Je m'efforcerai de présenter chacun de ces trois aspects de la réalité en les séparant les uns des autres d'une manière quelque peu artificielle mais nécessaire pour clarifier les enjeux. Mon point de départ sera l'année 1954 qui marque une première prise de conscience de la nécessité de définir une politique globale de restauration. Je choisirai comme point d'aboutissement le début des années 1990, marqué en 1993 par la publication d'un numéro spécial de la Revue générale des chemins de fer et en 1994 et par un bref bilan dressé par la SNCF.

\section{Une activité « structurellement déficitaire »}

La doctrine constante des dirigeants de la SNCF fut que la restauration était une activité "structurellement déficitaire " mais qu’elle engendrait une " induction de trafic » qui justifiait son maintien sur les grandes lignes. Cette position se trouve exprimée dans tous les rapports internes. En mars 1954, Charles Boyaux, directeur général de la SNCF, indiquait devant son conseil d'administration qu'il était nécessaire " de fournir aux voyageurs des grandes lignes des repas à prix normaux » car il fallait tenir compte de "l'intérêt commercial que présente la restauration pour les administrations des chemins de fer $\aleph^{2}$. De même Roger Guibert, directeur général adjoint, affirmait-il en 1963 que « le chemin de fer devait être en mesure de donner au public l'assurance qu'il pourra pendant le voyage se restaurer dans le train sous une forme ou sous une autre $»^{3}$.

L'histoire des concessions du service de restauration entre 1954 et 1994 se divise en trois périodes. De 1954 à 1961, de 1961 à 1972 et de 1972 à 1994.

2- Centre des archives historiques de la SNCF, Le Mans (désormais : CAH SNCF), 45LM639, Annexe au procès-verbal de la séance du conseil d'administration [de la SNCF] du 10 mars 1954. 3- CAH SNCF, 45LM535, Annexe au procès-verbal de la séance du conseil d'administration [de la SNCF] du 25 mars 1964. 


\section{4-1961}

De 1954 à 1961, les accords conclus en 1939 entre la CIWL et la SNCF permirent à la SNCF de faire porter la charge du déficit de la restauration sur le service des wagons-lits, qui était assez fortement excédentaire. La CIWL disposait d'un quasi-monopole. La SNCF avait pris le parti d'offrir à la clientèle des grandes lignes des repas et un service d'une qualité analogue à celle de l'offre d'un bon restaurant de province alors que le coût des prestations ferroviaires était supérieur de 50 à $60 \%$ à celui du restaurant en ville. Le contrat de 1939 établissait un prélèvement par paliers au taux progressif de 20, 40 et $60 \%$ indexé sur les variations des tarifs. La SNCF désignait les trains qu'elle désirait voir dotés d'un wagon-restaurant et la CIWL ne pouvait supprimer un service déficitaire. Dès 1954, cet équilibre était devenu fragile en raison de la nécessité pour la SNCF et la CIWL de faire face à la concurrence de l'avion et de réduire la tarification des wagons-lits. Le financement de la restauration ne pouvait plus être assuré «par le jeu de la compensation de ses déficits par les excédents des wagons-lits" (Charles Boyaux).

Charles Boyaux présenta au conseil deux propositions élaborées par un groupe de travail européen : le rachat du matériel CIWL, la désolidarisation des comptes des wagons-lits de ceux des wagons-restaurants. Elles furent rejetées. Un programme de redressement des comptes fut mis au point. Il prévoyait une coopération financière qui devait permettre à la CIWL d'enrichir son parc. La CIWL recevait une aide si le résultat d'exploitation était inférieur à l'annuité nécessaire à ce renouvellement.

Face à des résultats toujours décevants au cours de la décennie 19541964, un nouveau contrat entre la SNCF et la CIWL fut mis en place à la fin des années 1950. Il subordonnait le renouvellement du matériel à l'aide apportée par la SNCF à la CIWL. La SNCF rachetait le parc des voitures-restaurants et prenait à son compte la fourniture et l'entretien de ce matériel. Enfin la SNCF prélevait une redevance de $14 \%$ sur le bénéfice des voitures-lits et s'engageait à verser à la CIWL $80 \%$ de l'insuffisance éventuelle de son produit d'exploitation par rapport à ses annuités. Les services déficitaires pouvaient être supprimés.

\section{1-1972}

De 1961 à 1972 la mise en relation des annuités de renouvellement d'un matériel devenu vétuste et de l'aide apportée au service de restauration fut maintenue. Mais une réflexion, associée à une expérimentation permanente, fut entreprise. En mars 1967 une réforme radicale fut engagée après que La CIWL eut dénoncé les dispositions contractuelles la liant à la SNCF. Un 
nouveau contrat fut négocié qui abolissait la solidarité entre les comptes des wagons-restaurants et ceux des wagons-lits. La CIWL supportait le risque total de ses services de restauration (fig. 1). Si son exploitation dégageait un profit supérieur à $5 \%$, la SNCF participait à son partage. En revanche la SNCF indemnisait la CIWL si le déficit était supérieur à $25 \%$, en prenant à sa charge $75 \%$ du déficit excédant les $25 \%$. Le but de ce dispositif était d'aider la CIWL à financer les services créés récemment ou la mise en route de nouveaux trains rapides. Cet engagement se révéla désastreux pour la SNCF. Les charges engendrées par ce dispositif s'élevèrent à 0,21 million en 1967 et 18,7 millions en 1972. Si l'on y ajoute le coût de traction, d'entretien et d'amortissement du matériel spécialisé pris en charge par la $\mathrm{SNCF}$, le chiffre atteignait 71 millions de francs.

Figure 1 •

L'addition de deux clients de la CIWLT pour un repas rapide, plat, boisson et sans doute dessert, ticket de caisse, 17 septembre 1971. AHICF/coll. Jacques Hardel.

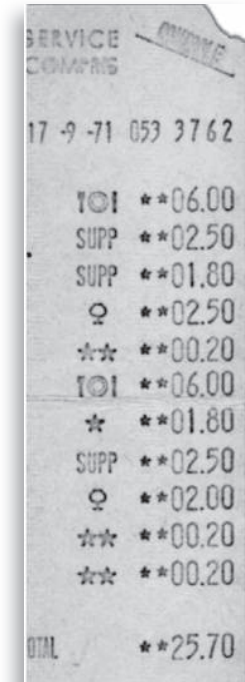

C.I.W.L.T. veus ramarcio

Deux orientations furent alors choisies : adopter des formules de restauration adaptées à la demande réelle, tenter l'expérience de la concurrence. En 1973, certaines parties du réseau furent concédées à quatre sociétés autres que la CIWL, à la suite d'une procédure de négociation de gré à gré :

- la CESAF, filiale de Servair, elle-même filiale d'Air France, obtint l'exploitation de l'étoile de Lyon.

- La société SHR, filiale d'une société qui exploitait les services de restauration maritimes vers la Corse, obtint divers services au départ de Nice.

- La société Rail-Service obtint toutes les relations au départ de Paris-Montparnasse.

- La société Gorsse formée par l'exploitant du buffet de ClermontFerrand reçut l'exploitation de la ligne Paris-Clermont.

Ces quatre sociétés employaient au total 553 agents. La CIWL, qui employait 1959 agents, conserva le reste du réseau, soit $83 \%$ de sa longueur. La SNCF accepta de participer aux dépenses de personnel de la CIWL à hauteur de $50 \%$ pour les wagons-restaurants et de $25 \%$ pour les gril express. 
En 1979 SHR reprit Rail-Service et, en 1980, abandonna son exploitation qui fut reprise par une filiale de la CIWL. Le TGV Sud-Est fut attribué dès 1981 à une autre filiale. La procédure de l'appel d'offres fut adoptée pour l'attribution du réseau est. Mais «il était bien entendu, indique un rapport de 1976, que le mieux disant des appels d'offres ne sera pas nécessairement retenu ».

Les contrats entre la SNCF et les concessionnaires reposaient sur le versement de subventions et de redevances calculées à partir des comptes. Les aides financières étaient définies en fonction de résultats établis service par service. Dans le cas de la CIWL, la SNCF versait une dotation destinée à couvrir les charges du personnel roulant et les prestations de nettoyage et d'entretien. Elle couvrait les déficits éventuels des différents services. Elle recevait en échange des redevances des services bénéficiaires. Les résultats d'exploitation étaient ainsi partagés. À partir de 1979, par exemple, la SNCF versa une subvention définie en fonction du résultat d'exploitation budgétisé, augmenté d'une rémunération de $3 \%$. Enfin, elle définissait la politique de restauration, faisait construire et entretenir le matériel et déterminait les programmes des services à mettre en place.

\section{2-1994}

À partir de 1972 le monopole de la CIWL fut donc aboli. Le but recherché était de faire jouer la concurrence et de réaliser les réformes reconnues nécessaires. Entre 1972 et 1994, trois tendances majeures se sont affirmées.

Les contrats, tout d'abord, sont accordés à la suite d'une procédure d'appel d'offres à laquelle répondent le plus souvent la CIWL, absorbée par Accor en 1991, et Servair ou leurs filiales. Les concessions avaient une durée limitée et la répartition des services était constamment modifiée. En 1985 l'exploitation de la restauration sur le Sud-Ouest fut retirée à la CIWL et confiée à Servirail, filiale de la SNCF et de la Cesaf. En 1987 Servair fut choisie pour assurer la restauration sur le TGV Atlantique. En 1993 un appel d'offres attribua au groupe Servair 60,5\% du chiffre d'affaires total de la restauration et à la CIWL $35 \%$.

Le milieu de la restauration ferroviaire s'est structuré. Le groupement des entreprises de la restauration ferroviaire (GERF), lancé en 1983, a été chargé de gérer la convention collective nationale de 1985. La Société anonyme de restauration ferroviaire (SARF), lancée en 1985, a pris en charge les problèmes généraux de coordination des activités des différentes sociétés. La même année a été créé le Comité communication et promotion, chargé de suivre le niveau de satisfaction des voyageurs. Enfin la société extérieure CREA a été chargée d'assurer le contrôle de qualité. Une autre forme de coopération 
a été la création de filiales communes pour gérer les centres d'avitaillement ouverts dans les grandes gares.

Les contrats sont caractérisés par le maintien d'une aide de la SNCF consacrée à la prise en charge totale ou partielle du déficit. Car la restauration restait «structurellement " déficitaire, nous l'avons dit. En 1994, le déficit de la restauration était de $56 \%$ du chiffre d'affaires alors que la restauration en ville réalisait un bénéfice de $10 \%$. L'une des causes de ce déséquilibre fut que les tribunaux ont imposé aux nouvelles sociétés l'obligation de reprendre le personnel de la CIWL en maintenant son statut. Les relations sociales étaient très mauvaises dans la plupart des sociétés. On peut comprendre dès lors que l'aide apportée par la SNCF ait pris la forme, très souvent, d'une participation aux charges de personnel. Par ailleurs la rémunération du concessionnaire est fixée en fonction de critères variés et changeants, comme les engagements assumés, les résultats obtenus dans le domaine de la qualité ou la participation à des " programmes qualité » contractuels.

\section{La recherche de la « formule " idéale pour le service}

En contrepartie des conditions avantageuses obtenues par la CIWL en 1954 l'entreprise s'est engagée à tout faire pour réduire le déficit de la restauration. Un bilan des résultats portant sur la période 1938-1962 a été dressé par Roger Guibert en 1962. Il permet de décrire la répartition des différentes formules de restauration à ces deux dates ${ }^{4}$. La comparaison entre les résultats de la période 1938-1954 et la période 1954-1962 n'était pas encourageante. De 1938 à 1954 le nombre des voyageurs-kilomètres sur les grandes lignes s'était accru de $25 \%$ et le nombre des repas distribués de $33 \%$. En revanche, de 1954 à 1962 le nombre des VK avait augmenté de $36 \%$ et le nombre des repas de type classique servis en wagons-restaurants avait baissé de $5 \%$. Il était passé de 2,7 millions en 1954 à 2,1 en 1962. Les causes de cette évolution étaient facilement identifiables, selon Guibert : la fermeture du service sur quelques lignes déficitaires, la création de modes de restauration plus économiques et l'accélération des trains sur plusieurs grandes lignes. Elle avait entraîné l'augmentation du nombre des voyages sans que le voyageur éprouve le besoin de se restaurer pendant le parcours. "Il y a une sorte de contradiction, constatait le 6 novembre 1968 le même Guibert, alors directeur général, entre l'équilibre de la restauration et la politique de développement des trains rapides que nous poursuivons ${ }^{5}$. En 1963 le pourcentage de voyageurs fréquentant les wagons-

4- Ibid.

5- CAH SNCF, 45LM600, Conseil d'administration du 6 novembre 1968. 
restaurants dans les trains drapeaux était de $25 \%$ mais de seulement $5 \%$ dans les trains rapides ordinaires.

Après le rachat du parc de la CIWL, la SNCF fit un considérable effort de renouvellement du matériel. Elle disposait de 114 wagons-restaurants. Un programme de radiation de 37 wagons-restaurants, d'achat de 42 unités neuves et de modernisation de 49 unités du matériel conservé fut entrepris. En 1971 l'âge moyen du matériel était de 16,5 ans au lieu de 33,3 en 1964. Le programme prévoyait la construction de wagons-restaurants affectés aux "grands trains d'affaires » et la modernisation du parc existant pour assurer le service ordinaire, soit en restauration classique, soit en restauration simplifiée.

Des formules nouvelles de restauration simplifiée furent expérimentées à l'occasion de ces commandes qui furent livrées à partir de 1965. Cette politique n'était pas nouvelle, mais elle fut intensifiée. Dès avant 1954 la formule de la voiture buffet, apparue dans les années 1930, avait rencontré un certain succès. Il s'agissait de voitures mixtes, une partie étant occupée par des compartiments ordinaires, une autre par une cuisine et une salle de restauration. Elles étaient exploitées dans les mêmes conditions que les wagonsrestaurants. Ces voitures ont assuré la restauration de 169000 voyageurs en 1954. De même, des repas étaient servis à la place sur les trains automoteurs. Cette formule concernait 87700 voyageurs en 1954. Les voitures nouvelles, de type snack bars ou bars, étaient divisées en deux parties, l'une comprenant des compartiments ordinaires, l'autre un office pour des plats simples, conserves réchauffées ou salades, le service étant assuré par deux agents. Elles avaient été essayées sur Paris-Strasbourg et " cette clientèle de choix s'est contentée d'un service très simplifié ", constatait Guibert. Il concluait que la formule était bonne pour les trains à fréquentation suffisante sans wagons-restaurants ni voitures buffets. Mais la formule du repas complet sur plateau servi à la place avait été abandonnée sur les services réguliers en raison des aléas de la vente et des problèmes d'hygiène qu'elle posait (fig. 2). Une formule plus originale fut testée sur des trains de standing moyen (trains 1109 et 1110 de la ligne du Bourbonnais) en mai 1963. Le wagon-restaurant était divisé en deux parties. Les voyageurs d'un premier service étaient admis dans une demi-salle, où ils étaient questionnés sur la boisson de leur choix et servis par plateaux. Pendant qu'ils consommaient, les mêmes opérations étaient effectuées dans l'autre demi-salle et pendant que ces voyageurs consommaient, ceux de la première demi-salle réglaient leur note et rejoignaient leur place. L'essai, selon le service commercial, fut réussi sur le plan technique. On avait pu accueillir 90 voyageurs dans une salle de 48 places et mobilisé trois agents au lieu de huit. Il fit pourtant l'objet de "réclamations vigoureuses " de la clientèle. 
Roger Guibert explique que " ces réclamations proviennent à coup sûr de personnes d'un certain âge qui exigent de faire des repas copieux avec un grand service dans les wagons-restaurants de nos trains ». Mais, dans dix ou quinze ans, «lorsque la majorité des réclamants actuels aura disparu, la SNCF pourra alors appliquer la formule simplifiée $»^{6}$. Une autre formule, celle du self-service, fut expérimentée sur le Gallia express dans une voiture divisée en deux salles. De même, 90 voyageurs pouvaient y être servis en une heure et elle mobilisait trois agents au lieu de huit. Guibert affirmait qu'une " fraction très importante des voyageurs demandera instamment des menus plus simples, moins chers avec un menu très simplifié, ce qui entraînera une augmentation $\mathrm{du}$ rendement des wagons-restaurants. Mais il faudra choisir entre des demisalles en self-service et des wagons-restaurants modifiés ». Il fallait cependant maintenir les wagons-restaurants pour assurer les "grands trains d'affaires". Les voitures buffets et les voitures snack-bar furent réaménagées à l'occasion des grandes opérations d'entretien. Leur aménagement " ne posait pas de problèmes très importants car il était facilement obtenu à partir de voitures ordinaires $»^{7}$.

Figure 2 เ

Le menu et les prix du plateau servi à la place, une des formules de la vente ambulante, octobre 1959. AHICF/coll. Jacques Hardel.

\section{6- Ibid.}

7- Ibid.

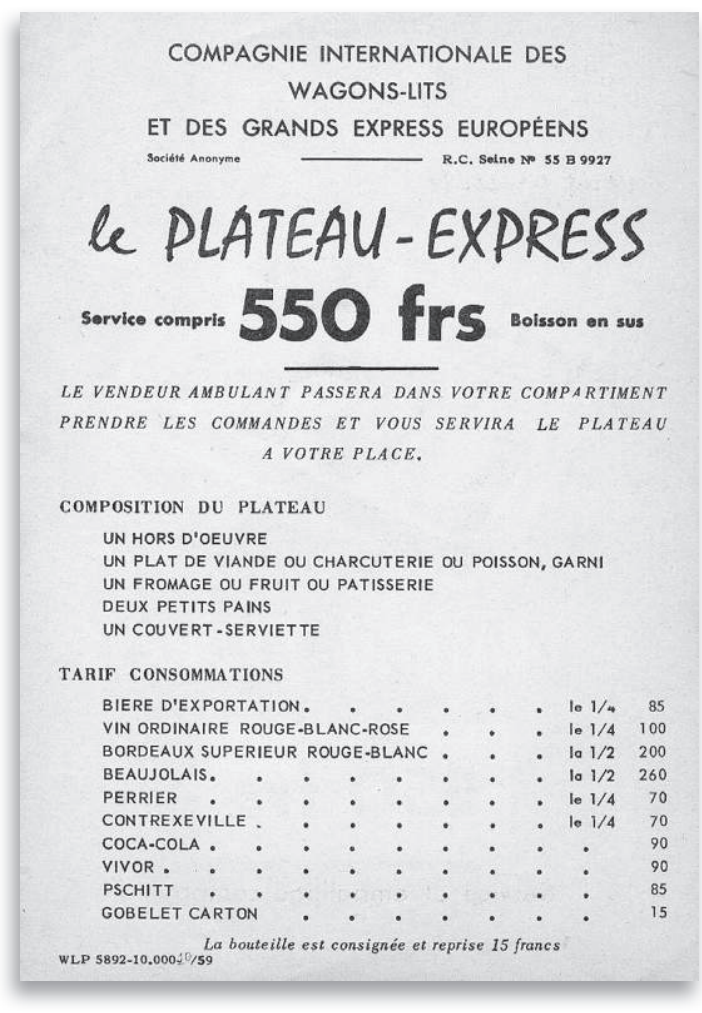


Un bilan financier des différents secteurs fut établi, grâce à l'adoption des méthodes comptables et de contrôle de gestion modernes. Il mettait en valeur la grande diversité des résultats des différents services. La réforme de 1972 fut suivie d'une enquête très approfondie, portant sur la qualité des services et les résultats financiers entre 1973 à 1976 des différentes sociétés prestataires qui venaient d'être mises en place. Les rapports dressant le bilan de ces enquêtes analysent les résultats obtenus par les gérants et forment autant de comptes rendus d'expériences réalisées par ces sociétés. Ces années marquent ainsi un tournant décisif de l'histoire de la restauration ferroviaire. Les rapports détaillés rédigés par les contrôleurs ont nourri la réflexion portant sur la comparaison des différentes formules. De plus, c'est alors que furent mises en service les premières voitures Corail qui permettaient d'organiser un service à la place sans difficulté majeure, mais qui exigeaient elles aussi une expérimentation.

L'un des critères appliqués pour apprécier la qualité du service fut la capacité à exploiter en utilisant les nouveaux types de restauration et à assurer le service en voiture Corail ou en TGV. C'est ainsi que Servair, malgré une gestion jugée très mauvaise, fut retenue. Car il y avait un lien " entre cette exploitation et les études de restauration Corail et TGV ». Le savoir-faire aérien était transposable sur ces deux matériels. De même, le réseau SHR permit de tester des formules nouvelles pour organiser la vente ambulante et le service de gril express. Il réalisa un démarrage correct de la restauration Corail qui devait remplacer le service snack. Le gril express fut exploité par les trois opérateurs du Sud-Est (fig. 3). La Cesaf enfin, après avoir échoué à exploiter un service de plateaux dans des rames qui ne disposaient pas de tablettes et de portes automatisées, s'orienta vers une " exploitation expérimentale ". "On a cherché, indique un rapport de Paul Gentil, directeur général, en 1975, à développer un type de service qui permettra de résoudre la principale source de coûts en faisant appel à la préparation à terre et au service en voitures non (ou peu) spécialisées. Le fait de détacher le personnel roulant des tâches de production a pour objectif d'éviter le recours aux " professionnels ", de rénover et de réduire les effectifs et les qualifications du matériel roulant ${ }^{8}$. " De plus un certain désordre dans l'organisation (services non assurés, approvisionnements interrompus, mal prévus) a été compensé dans le jugement des voyageurs, portant sur l'accueil et l'ambiance et, aussi, sur la qualité du personnel. Des plateaux-repas ont remplacé le service du type wagon-restaurant. Une tentative d'extension à la deuxième classe a été organisée. C'est ainsi que

8- CAH SNCF, 45LM698, Conseil d'administration du 4 juin 1975. 
naquit la restauration moderne. Elle fait la synthèse des expériences réalisées par les différentes sociétés entre 1973 et 1976 pour évoluer ensuite assez rapidement (fig. 4).

En 1975, le rapport de Gentil misait sur le développement des voitures gril express dont la capacité de restauration, selon lui, était deux à trois fois plus grande que celle de la voiture classique, ce qui correspond à un fréquentation voisine du double. Dès lors, la formule d'emploi la plus favorable " consiste à associer un gril express à une voiture dont les occupants peuvent se faire servir à leur place ». Cette prévision ne se confirme qu’à moitié. Au lieu de la combinaison gril express - service à la place c'est la formule bar et service à la place fonctionnant séparément qui s’est imposée. En 1994, aussi bien en matériel classique qu'en matériel TGV, l'essentiel du parc se compose de voitures bars accessibles aux voyageurs de première et seconde classe et de voitures de restauration à la place accessibles aux voyageurs de première classe. Quelques autres séries très limitées sont appelées à disparaitre. Les voitures bars se différencient en fonction de la nature du train : la moitié d'une voiture sur les trains Corail, les deux tiers sur le TGV Sud-Est.

Figure 3

La formule « gril express » proposée par la CIWL, feuillet publicitaire, années 1970. AHICF/coll. Jacques Hardel.

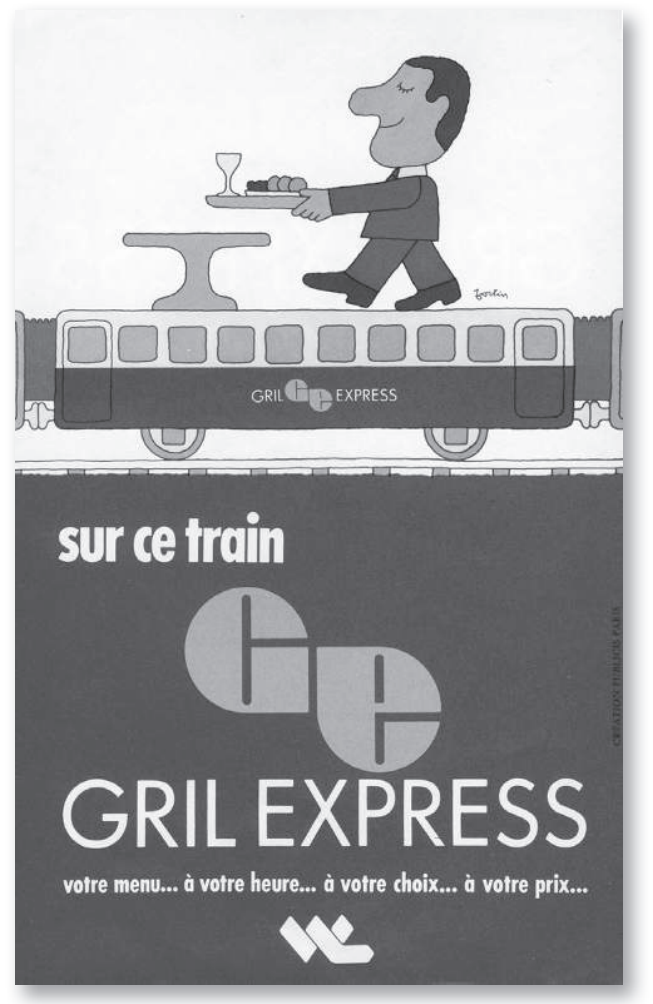




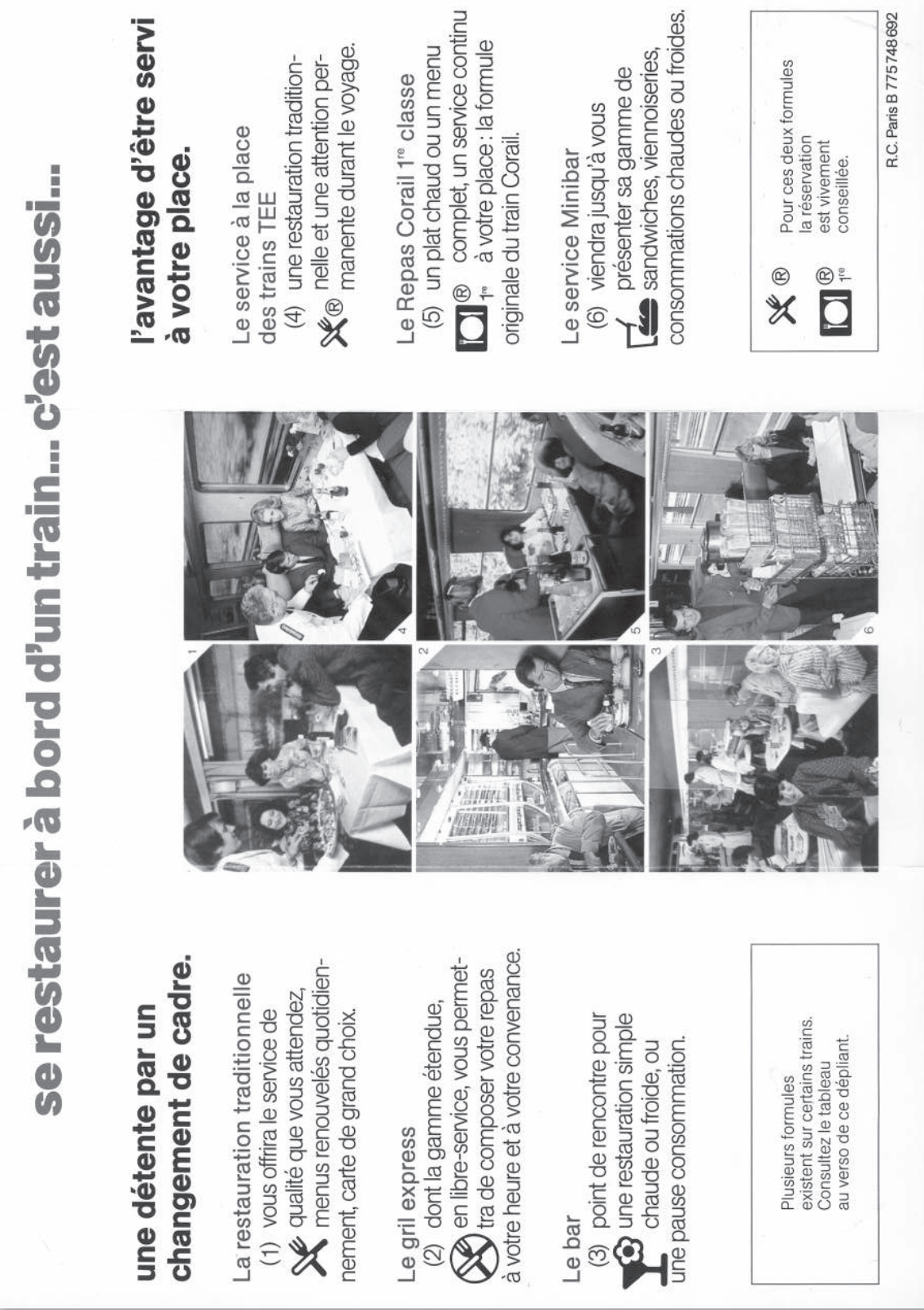

- Figure 4. Dépliant d'information pour la restauration « Corail », incluant l'offre « traditionnelle » des wagons-restaurants, années 1970. AHICF/coll. Jacques Hardel. 


\section{Des repas adaptés aux services et aux attentes de la clientèle}

En 1954 le produit phare de la CIWL est le repas copieux d'un très bon standing servi en wagon-restaurant. Cette formule fut dès le début des années 1950 menacée de disparition du fait du déficit important qu'elle engendrait. Charles Boyaux dans son rapport de 1954 envisage la possibilité de remplacer les wagons-restaurants par des voitures buffets ou des services de vente ambulante. Cette solution est aussitôt rejetée au profit du nouveau contrat que nous avons décrit plus haut associé à une recherche d'économies dans l'exécution même du service des wagons-restaurants en accord avec la CIWL. "Il est en effet permis de concevoir des formules de repas (service froid sur plateau, comme dans les avions ou plat unique...) exigeant des frais de préparation ou de service moins élevés que les menus actuels. "

Cette offre "traditionnelle " était caractérisée par un bon standing et un prix de repas élevé. Le service était très onéreux en personnel. Son coût, nous l'avons dit, était de près de $60 \%$ supérieur à celui d'un bon restaurant de province et ne cessait de croître. Déjà sur les trains automoteurs et sur Paris-Bruxelles la formule du repas classique à la place avait été proposée, les voitures étant des voitures à couloir et cuisine. La formule de la voiture buffet rencontrait toujours un certain succès : le nombre des repas servis dans ces voitures buffets était passé de 169000 à 278000 et dans les trains automoteurs de 87700 à 240000 entre 1954 et 1962. La vente ambulante, apparue en 1950 , avait connu une croissance très rapide : les recettes engendrées par cette prestation étaient passées de 2,3 millions de francs en 1954 à 9 millions en 1962, soit $7 \%$ et $13 \%$ des recettes totales. Entre 1954 et 1961 de nombreuses expériences furent testées pour orienter l'offre vers l'allégement des repas. Dans les wagons-restaurants on proposa des " menus allégés ", introduits en 1961. C'est un menu classique dans lequel on a supprimé un plat, l'entrée en général. Cette solution eut un grand succès. Les menus allégés représentaient en $196330 \%$ des repas servis dans les voitures proposant les deux formules. Mais cette solution n'est pas rationnelle et n'engendre ni économie ni une plus grande fréquentation. La formule du paquet repas rencontra elle aussi un grand succès après 1962, sans que l'expérience soit poursuivie par la suite.

S'interrogeant en 1963 pour savoir « quel type de repas faudra-t-il servir dans les wagons-restaurants et sous quelle forme ", Roger Guibert répondait qu'il fallait prendre en considération l'évolution générale des habitudes des Français depuis un certain nombre d'années. "On mange, disait-il, moins copieux et les restaurants à prix fixe et à menu copieux tendent à disparaître au bénéfice d'établissements offrant un service très simple, self-service souvent. " 
Les buffets des grandes gares, comme Lyon-Perrache par exemple, appliquaient cette formule, qui remportait un grand succès.

Lors de la réforme de 1967, $5 \%$ seulement des voyageurs faisaient appel au service de restauration dont $1,5 \%$ aux wagons-restaurants et 3,5\% aux ventes ambulantes. Leur succès ne faiblissait pas. Le coefficient de fréquentation des wagons-restaurants pendant les plages horaires correspondant aux heures de repas normaux était de $12 \%$ sur les wagons-restaurants et de $30 \%$ pour les ventes ambulantes. Sur Bruxelles-Paris, entre $12 \mathrm{~h}$ et $14 \mathrm{~h}$ la première de ces fréquentations atteignait $60 \%$. Il fallait envisager de remplacer complètement ou partiellement des wagons-restaurants par des voitures buffets, rechercher des économies dans l'exécution du service en appliquant de nouvelles formules et prendre acte de "l'inadaptation croissante des prestations aux demandes de la clientèle ", que les nombreuses enquêtes de marchés confirmaient. C'est ainsi que les voyageurs de première classe se plaignaient d'être obligés " de supporter la dépense d'un repas, dont le volume et le degré d'élaboration allaient nettement au-delà de leurs désirs ».

Il fallait, selon Guibert, mettre au point des services " simplifiés » dont " le but sera de nourrir simplement les voyageurs avec un service rapide et à un prix raisonnable, ce qui permettra d'augmenter le débit des wagons-restaurants et de satisfaire une fraction plus importante de la clientèle du train sans accroître les moyens matériels - voitures et personnels - et, pensonsnous, en améliorant le bilan ». La technique des plats surgelés "s'articulait très bien dans cette évolution ». Elle permettait de réduire les dépenses élevées de personnel spécialisé, particulièrement difficile à recruter pour les services supplémentaires d'été. Des études prometteuses ont été menées à la gare SaintLazare. Mais il est nécessaire d'ajouter à ce service " une chaîne des restaurants à poste fixe ». Il faudra donc de longs délais avant que cette solution puisse être mise en application. Dans les années 1980 la surgélation, qui était le complément de la préparation des repas à l'extérieur des voitures, se trouva ainsi placée au cœur du système. Elle prolongeait un effort de rationalisation des achats de denrées et des approvisionnements qui date des années 1960. Il entraîna une forte réduction des dépenses.

En 1994 la restauration concernait 15 millions de consommateurs et 1300 trains de grandes lignes, soit un consommateur pour cinq voyageurs. Son organisation repose sur deux métiers : le service à bord et l'avitaillement chaque jour, le long de la chaîne de froid, de tous les trains en partance depuis le commissariat hôtelier qui élabore les repas jusqu'au chargement du train en passant par l'espace de stockage et les camions portant les cellules isothermes. Hors région parisienne, huit centres occupaient moins de $1000 \mathrm{~m}^{2}$, 
deux en région parisienne. Deux centres en province occupaient entre 1000 et $2000 \mathrm{~m}^{2}$ et deux en région parisienne. Deux centres de plus de $5000 \mathrm{~m}^{2}$ situés en région parisienne avaient une activité de production. En 1994 les petits services d'avitaillement (SAV) étaient en cours de disparition au profit de centres plus grands. Un accord était en cours de négociation pour assurer le partage des coûts des SAV. De plus la vente ambulante a été réorganisée pour obtenir un service mieux ciblé et une organisation plus souple. Les voitures bar et les autres formules de "solutions simplifiées " sont complémentaires du développement de la vente ambulante centrée sur le sandwich et la bière. Une statistique fondée sur le nombre de courses donne les résultats suivant : $57 \%$ des consommateurs utilisent les bars, $21 \%$ la vente ambulante, $16 \%$ la restauration sur place et $6 \%$ d'autres formules. La répartition des recettes est très différente (fig. 5).

Figure 5 •

L'éventail des services de restauration proposées par la SNCF, feuillet publicitaire, après 1985 et avant 1992. AHICF/coll. Jacques Hardel.

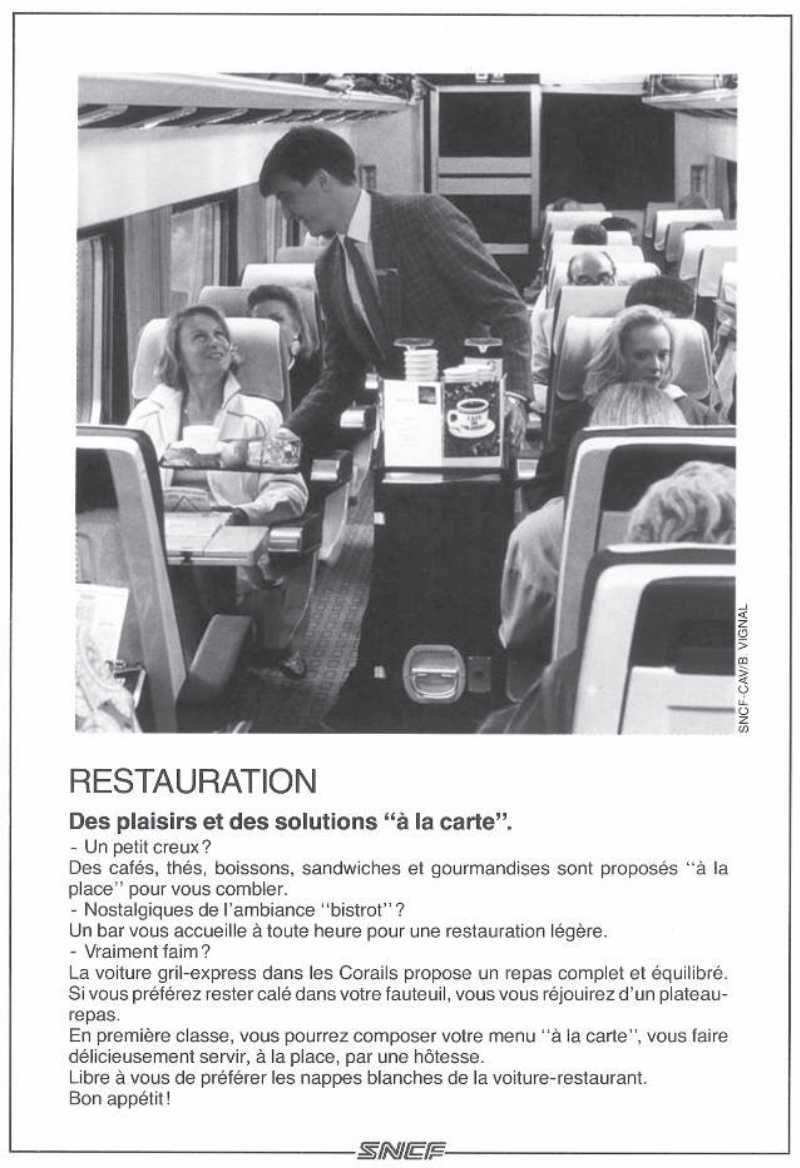


L'ensemble de ces observations débouche sur trois constats. La relation entre la SNCF et ses concessionnaires ne s'est transformée que lentement. Mais le pas décisif est contemporain d'une période de transformation radicale des techniques, des stratégies et des structures de l'entreprise. L'histoire de la restauration est concrètement inséparable de celle de l'organisation de l'espace dans les voitures et du triomphe de la voiture coach.

Elle est étroitement dépendante de l'évolution des modes d'exploitation, de la politique des vitesses et enfin de la transformation radicale des relations entre la SNCF et ses partenaires.

La restauration s'est adaptée, comme les autres fonctions, à un changement profond de "l'environnement " économique et social et des pratiques alimentaires. Pourtant il a fallu du temps pour que soit abandonnée la formule du wagon-restaurant classique. Mais la SNCF s'est finalement soumise aux changements des modes de vie, selon une logique générationnelle. Le wagonrestaurant renaîtra, à n'en pas douter, de ses cendres sous la pression de nouveaux consommateurs friands de repas de qualité mais non pas nécessairement « copieux ».

Il faudra écrire l'histoire du plateau comme celle du sandwich, comprendre les raisons du succès du gril express et de la voiture buffet ou encore le triomphe du bar... Il faudra retracer l'histoire des progrès réalisés par les techniques du froid comme celle des exigences de plus en plus rigoureuses en matière d'hygiène alimentaire. 